\title{
Axial temperatures and electron densities in a flowing cascaded arc: model versus experiment
}

\author{
J J Beulens, $M$ J de Graaf and D C Schram \\ Eindhoven University of Technology, Department of Physics, \\ PO Box 513, $5600 \mathrm{MB}$ Eindhoven, The Netherlands
}

Received 8 September 1992, in final form 16 February 1993

\begin{abstract}
A Fabry-Pérot interferometer is used to measure argon ion line profiles in a strongly flowing cascaded arc plasma. Heavy-particle temperatures, electron densities, and electron temperatures are derived from the Doppler width, the Stark width and the line/continuum ratio respectively. The electron temperatures are also obtained from the electrical conductivity of the plasma. The plasma parameters along the arc channel are values averaged over the arc cross section and are determined with an accuracy of $2-5 \%$. Comparison of the measurements with model calculations is also made, to improve understanding of the plasma processes in the arc channel.
\end{abstract}

\section{Introduction}

Since about 1985 a cascaded arc has been used as a particle source in the deposition set-up described by Kroesen [1]. This method of deposition has proved to be very fast and efficient for growing carbon films, varying from graphite and diamond to polymers $[1,2]$. The most important difference of this method, with respect to $\mathrm{RF}$ techniques, is that the three most important functions of a plasma deposition process, namely dissociation and ionization, transport, and deposition are spatially separated. The separation makes it possible to optimize the process functions step by step independently. Dissociation takes place in a cascaded arc burning on argon. The temperatures in the arc are about $10000-12000 \mathrm{~K}$. At the end of this arc, hydrocarbons are injected which are dissociated and ionized effectively. In this region the plasma expands supersonically into a vacuum vessel. This means that the plasma cools and the formed hydrocarbon fractions are transported towards the substrate, where a carbon film can grow. The quality of the film depends mainly on the amount of energy available for each injected carbon atom $[1,3,4]$. The energy available per deposition event comes from excited and ionized argon particles that are produced inside the cascaded arc. Therefore the cascaded arc is investigated numerically and expermentally in order to improve the ionization efficiency and to control the parameters of the plasma flowing out. The conservation laws for mass, momentum and energy for both the electrons and the heavy particles are solved two-dimensionally by a numerical method with a non-staggered grid [5]. With
Fabry-Pérot interferometry, spectral lines are measured which yield values for heavy particle and electron temperatures and electron density as a function of the axial position in the cascaded arc. The experimental results obtained by the optical diagnostics are compared with the numerical data of the two-dimensional model. The electron temperatures are also calculated from the plasma potential along the arc channel by using theoretical values of the plasma conductivity as a function of electron temperature. For the present work it is also necessary to know the argon ion fluence, emerging from the plasma source, which contains the chemical energy used to dissociate and ionize monomer fragments.

\section{Model}

Significant experimental and theoretical research has been performed on the cascaded arc. In the domain of modelling several one-dimensional models have been developed $[1,6,7]$. However, it has been recognized that two-dimensional modelling of the processes in plasma torches is needed $[8,9]$, in order to treat correctly some phenomena such as highly non-isothermal flow, deviations from local thermal equilibrium (LTE), as well as diffusion and heat conduction. In this work the results obtained with a two-dimensional model by Beulens et al [5] are used and compared with experimental results.

The model will be described here in a condensed way. In the approach adopted, the plasma is treated as a twophase medium, which consists of heavy particles (neutral atoms and ions) and light particles (electrons). For a full 
description of this two-phase flow one needs to solve the mass, momentum and energy equations for both phases. A two-phase flow character is kept only with respect to the heavy particle and electron temperatures, which are allowed to be different and are described by separate energy (balance) equations. Ohmic heat input is consumed by electrons, whose temperature increases, and is dissipated in frequent elastic and inelastic collisions with the heavy particles, which are heated and ionized to a higher degree. The mass of the electrons is small and the elastic collisions are not always efficient enough to equalize the temperatures of the two phases, especially near the inlet regions of the flow, where the cold gas is introduced, and in the vicinity of cooled walls. The governing equations of the model are shown below: for details see [5].

\subsection{Flow equations}

The plasma fiow is described by the following continuity and momentum equations:

$$
\begin{gathered}
\frac{\partial}{\partial x}(\rho u)+\frac{1}{r} \frac{\partial}{\partial r}(r \rho v)=0 \\
\frac{\partial}{\partial x}\left(\rho u^{2}\right)+\frac{1}{r} \frac{\partial}{\partial r}(r \rho u v) \\
=2 \frac{\partial}{\partial x}\left(\mu \frac{\partial u}{\partial x}\right)+\frac{1}{r} \frac{\partial}{\partial r}\left(r \mu \frac{\partial u}{\partial r}\right) \\
+\frac{1}{r} \frac{\partial}{\partial r}\left(r \mu \frac{\partial v}{\partial x}\right)-\frac{\partial p}{\partial x} \\
\frac{\partial}{\partial x}(\rho u v)+\frac{1}{r} \frac{\partial}{\partial r}\left(r \rho v^{2}\right) \\
=\frac{\partial}{\partial x}\left(\mu \frac{\partial v}{\partial x}\right)+\frac{2}{r} \frac{\partial}{\partial r}\left(r \mu \frac{\partial v}{\partial r}\right) \\
+\frac{\partial}{\partial x}\left(\mu \frac{\partial u}{\partial r}\right)-\frac{2 \mu v}{r^{2}}-\frac{\partial p}{\partial r}
\end{gathered}
$$

in which $u$ and $v$ denote the axial and radial velocities, $\rho$ is the plasma mass density, $p$ is the pressure, $\mu$ is the dynamic viscosity, and $x$ and $r$ are the axial and radial coordinates.

\subsection{Plasma composition equations}

The model is formulated for a monatomic gas undergoing single ionization at locally quasi-neutral conditions. Therefore the plasma composition is defined by a single conservation equation: the electron continuity equation

$$
\begin{gathered}
\frac{\partial}{\partial x}\left(n_{\mathrm{e}} u\right)+\frac{1}{r} \frac{\partial}{\partial r}\left(r n_{\mathrm{e}} v\right)=\frac{\partial}{\partial x}\left(D_{\mathrm{a}} \frac{\partial n_{\mathrm{e}}}{\partial x}\right) \\
+\frac{1}{r} \frac{\partial}{\partial r}\left(r D_{\mathrm{a}} \frac{\partial n_{\mathrm{e}}}{\partial r}\right)+R_{\mathrm{ne}}
\end{gathered}
$$

where $D_{\mathrm{a}}$ denotes the ambipolar diffusion coefficient and $R_{\mathrm{ne}}$ is the electron source term, which coincides with the volumetric ionization-recombination rate.

\subsection{Energy equations}

The energy conservation equations are written both for the heavy particles and for the electrons. The heavyparticle energy equation is given in the form of conservation of the heavy-particle stagnation enthalpy

$$
\begin{array}{r}
h_{\mathrm{h}}=C_{P_{\mathrm{h}}} T_{\mathrm{h}}+\frac{1}{2} v_{\mathrm{t}}^{2} \quad v_{\mathrm{t}}^{2}=u^{2}+v^{2} \\
\frac{\partial}{\partial x}\left(\rho u h_{\mathrm{b}}\right)+\frac{1}{r} \frac{\partial}{\partial r}\left(r \rho v h_{\mathrm{h}}\right)=\frac{\partial}{\partial x}\left(\frac{\lambda_{\mathrm{h}}}{C_{P \mathrm{~h}}} \frac{\partial h_{\mathrm{h}}}{\partial x}\right) \\
+\frac{1}{r} \frac{\partial}{\partial r}\left(r \frac{\lambda_{\mathrm{h}}}{C_{P \mathrm{~h}}} \frac{\partial h_{\mathrm{h}}}{\partial r}\right) \\
+\frac{\partial}{\partial x}\left[\mu\left(1-\frac{1}{P_{\mathrm{r}}}\right) \frac{\partial \frac{1}{2} v_{\mathrm{t}}^{2}}{\partial x}\right] \\
+\frac{1}{r} \frac{\partial}{\partial r}\left[r \mu\left(1-\frac{1}{P_{\mathrm{r}}}\right) \frac{\partial \frac{1}{2} v_{\mathrm{t}}^{2}}{\partial r}\right]+Q_{\mathrm{eh}}
\end{array}
$$

where $C_{P \mathrm{~b}}=5 R_{\mathrm{h}} / 2$ denotes the specific heat of heavy particles, $\lambda_{\mathrm{h}}$ is the heavy-particle heat conductivity and $P_{\mathrm{r}}=\mu C_{P \mathrm{~h}} / \lambda_{\mathrm{h}}$ is the heavy-particle Prandtl number. The last term on the right-hand side of equation (6), $Q_{\text {eh }}$, is the net volumetric energy exchange rate between electrons and heavy particles due to elastic collisions.

The intrinsic electron energy equation has the following form:

$$
\begin{aligned}
\frac{\partial}{\partial x}\left(\frac{5 n_{\mathrm{e}} u_{\mathrm{e}} k T_{\mathrm{e}}}{2}\right)+\frac{1}{r} \frac{\partial}{\partial r}\left(\frac{5 r n_{\mathrm{e}} v_{\mathrm{e}} k T_{\mathrm{e}}}{2}\right) \\
=\frac{\partial}{\partial x}\left(\lambda_{\mathrm{e}} \frac{\partial T_{\mathrm{e}}}{\partial x}\right)+\frac{1}{r} \frac{\partial}{\partial r}\left(r \lambda_{\mathrm{e}} \frac{\partial T_{\mathrm{e}}}{\partial r}\right) \\
+u_{\mathrm{e}} \frac{\partial}{\partial x}\left(n_{\mathrm{e}} k T_{\mathrm{e}}\right)+v_{\mathrm{e}} \frac{\partial}{\partial r}\left(n_{\mathrm{e}} k T_{\mathrm{e}}\right)+Q_{\mathrm{ohm}} \\
\quad+Q_{\text {ion }}-Q_{\text {eh }}+Q_{\text {rad }}
\end{aligned}
$$

where $\lambda_{c}$ is the electron heat conductivity, $Q_{\mathrm{ohm}}$ is the energy source due to ohmic heating, $Q_{\text {ion }}$ is the energy sink for ionization of neutral atoms or source if ion recombination takes place, and $Q_{\mathrm{rad}}$ is the net volumetric radiative energy exchange rate. In this equation the velocities $u_{\mathrm{e}}$ and $v_{\mathrm{e}}$ are the electron velocities. These can be rewritten using the current density $j=n_{e} q\left(v_{i}-v_{\mathrm{e}}\right)$, in which $v_{i}$ is the ion velocity (and equal to the gas velocity), and $v_{\mathrm{e}}$ is the electron velocity.

The two-dimensional two-temperature model is developed for the predictions of a strongly flowing monatomic cascaded arc plasma. The flow is assumed to be axisymmetric, two-dimensional, compressible and strongly non-isothermal. The argon plasma is considered as singly ionized and locally quasi-neutral $\left(n_{\mathrm{e}} \simeq n_{\mathrm{i}}\right)$ with local temperature non-equilibrium $\left(T_{e} \neq T_{h}\right)$. The electric field is assumed to be one-dimensional and uniform over the arc cross section $\left(E_{\mathrm{r}}=0, \partial E_{\mathrm{x}} / \partial r=0\right)$. The total current is specified as an input parameter and is kept constant along the arc channel. A numerical grid with $23 \times 24$ non-uniformly distributed points is used for all calculations. The calculations are performed on an IBM PC/RT. 


\section{Experimental set-up}

The cascaded arc (figure 1) used in this work consists of a stack of ten water-cooled copper plates insulated electrically from each other by plastic (PvC) spacers and $\mathrm{O}$ rings, three tungsten-thorium cathodes at one end and an anode at the other. Through the copper plates and the anode plate there is a bore of $4 \mathrm{~mm}$ diameter, forming a cylindrical channel of length $60 \mathrm{~mm}$. The argon gas is injected through mass flow controllers at the cathode side. The gas or plasma is extracted through a nozzle in the anode plate into a low-pressure vessel. The pressure in the vessel is about 1 mbar, whereas the pressure in the arc is about 0.5 bar. The latter results in an acceleration of the plasma flow to sonic velocities at the arc exit, and subsequently a supersonically expanding plasma jet.

For the experiments, the cascaded arc has two special features. In each plate, side-on observation of the plasma is possible through smali quartz windows cemented in a narrow radial bore in each cascade plate. The diameter of this bore is chosen to be as small as possible to avoid disturbance of the gas flow pattern. This feature enables a radially integrated measurement to be made of the emitted light from the plasma. Secondly, the bore that contains the window is also connected to a pressure gauge. The pressure is measured by an MKS baratron pressure transducer.

The light emitted by the plasma is coupled out via an optical fibre and then coupled into an optical system

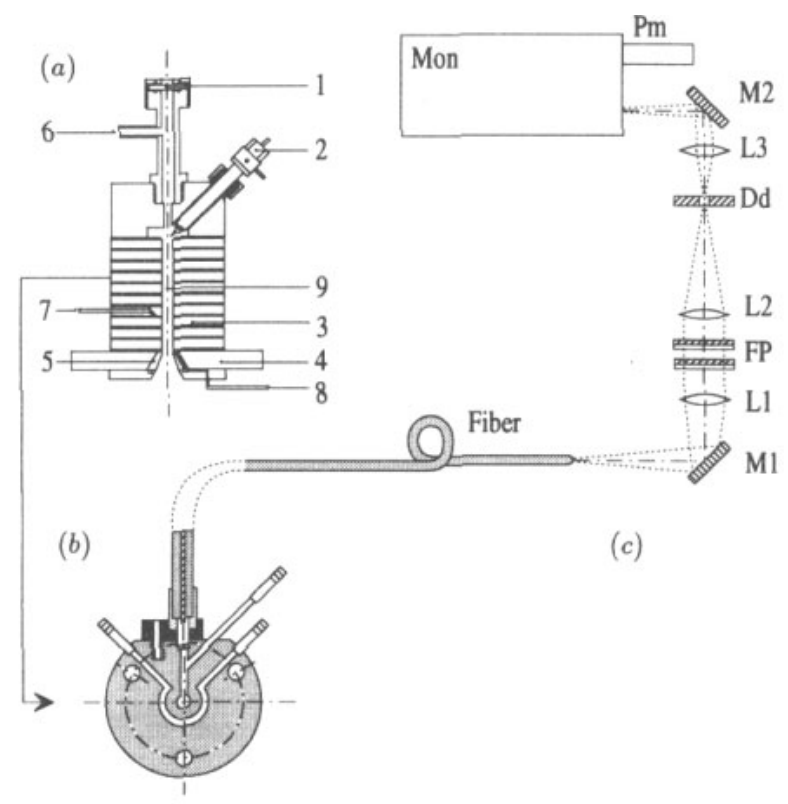

Figure 1. (a) Outline of the cascaded arc particles source: 1 , end-on viewing window; 2 , tungsten-thorium cathode $(3 \times)$; 3 , copper cascade plate $(10 \times, 4 \mathrm{~mm}$ bore); 4 , anode plate; 5 , anode nozzle; 6 , argon inlet; 7 , hydrogen inlet; 8 , hydrocarbon inlet; 9 , plasma channel. (b) Cascade plate with fibre optics and pressure meter channel perpendicular to the plasma channel. (c) The optical system consists of: fibre connected to each cascaded arc plate (see (b)); mirror M1; lens $L 1(f=400 \mathrm{~mm})$; Fabry-Pérot interferometer FP; lens $\mathrm{L} 2(f=400 \mathrm{~mm})$; diaphragm Dd $(d=1 \mathrm{~mm})$; lens L3 $(f=75$ $\mathrm{mm})$; mirror M2; a monochromator Mon, and a photomultiplier PM. (figure 1, right-hand side). Between the two lenses L1 and L2 the light beam is parallel and thus a Fabry-Perot interferometer can be mounted here. The light source is a hollow cathode arc or a glow discharge on argon, to make a wavelength reference and to measure the apparatus profile. Lens L2 images the exit of the fibre on diaphragm D1. Finally, lens L3 images the diaphragm on the entrance slit of the monochromator (Jarrell-Ash $0.5 \mathrm{~m}$ ). On the exit slit a photomultiplier is mounted. In the monochromator a $25 \mu \mathrm{m}$ slit and a $50 \mu \mathrm{m}$ slit are placed in order to obtain a trapezoidal monochromator apparatus profile with a flat part of $40 \mathrm{pm}$. The pressurescanned Fabry-Pérot interferometer in this experiment has a $1 \mathrm{~mm}$ spacing which gives a free spectral range (FSR) of $90 \mathrm{pm}$. The interferometer was placed in a thermo-statted box where the temperature was controlled at $(30 \pm 0.1)^{\circ} \mathrm{C}$. The gas pressure inside the FabryPérot was controlled by pneumatic valves in order to avoid heating of the argon filling gas by electric valves. Needle valves restricted the gas flow to achieve a slow increase or decrease of the pressure. In this way temperature changes caused by adiabatic compression and expansion respectively were avoided. By stanning the pressure in the Fabry-Pérot (1-4 bar argon, 1 FSR $\hat{=}$ 0.8 bar) a spectral line can be scanned. The apparatus profile has an FWHM of $1.5 \mathrm{pm}$. A typical linle profile is shown in figure 2 for $I_{\text {arc }}=50 \mathrm{~A}$ and $[\mathrm{Ar}]=50 \mathrm{scc} \mathrm{s}^{-1}$.

\section{Plasma diagnostics}

The wavelength-dependent intensity profile of a spectral line emitted by the plasma is determined by several physical effects. Each effect can result in a shift of the line position and/or a broadening of the line profile with usually either a Gauss- or a Lorentz-like contribution. In the cascaded arc studied in this paper, two broadening mechanisms are important: Doppler and Stark broadening.

Thermal motion of particles in the plasma gives rise to a Doppler broadening of spectral lines. Assuming a

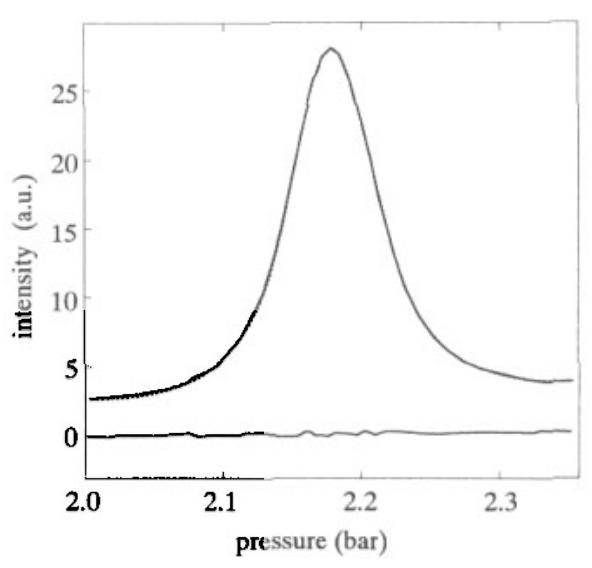

Figure 2. A typical measured (full line) line profile with a Voigt fit (broken line) and residue for $l_{\text {arc }}=50 \mathrm{~A}$ and $[\mathrm{Ar}]=50 \operatorname{scc~s}^{-1}$, as a function of the pressure in the Fabry-Pérot interferometer. 
Maxwellian velocity distribution for the emitting particles, a Gaussian line profile results. The quadratic Stark effect, caused by the influence of charged particles on the energy levels of the emitting atom or ion, gives a Lorentzian line profile $[10,11]$. We chose to use an Ar II line as for these lines the Doppler and Stark broadenings are of the same order of magnitude for the plasma we use.

Deconvolution of a measured line profile gives then the heavy-particle temperature from the Gauss width and the electron density from the Lorentz width.

Besides line emission also continuum emission caused by collisions between electrons and atoms (free-free electron-atom radiation), and electrons and ions (free-free electron-ion radiation (bremsstrahlung) and free-bound radiation). The free-bound and free-free continuum emissivities of argon can be expressed as follows $[12,13]$ :

$$
\varepsilon=\varepsilon_{\mathrm{fb}}+\varepsilon_{\mathrm{ff}}^{\mathrm{ei}}+\varepsilon_{f f}^{\mathrm{ea}}
$$

with

$$
\begin{gathered}
\varepsilon_{\mathrm{ff}}^{\mathrm{ej}}=\frac{C_{1}}{\lambda^{2}} \frac{n_{\mathrm{e}} n_{\mathrm{i}}}{\sqrt{T_{\mathrm{e}}}} \xi_{\mathrm{ff}}\left(\lambda, T_{\mathrm{e}}\right) \mathrm{e}^{-h c / \lambda k T_{\mathrm{e}}} \\
\hat{\varepsilon}_{\mathrm{ff}}^{\mathrm{ea}}=\frac{C_{2}}{\lambda^{2}} n_{\mathrm{e}} n_{\mathrm{a}} \sqrt{ } T^{3} Q\left(T_{\mathrm{e}}\right)\left[\left(1+\frac{h c}{\lambda k T_{\mathrm{e}}}\right)^{2}+1\right] \mathrm{e}^{-h c / \lambda k T_{\mathrm{e}}} \\
\varepsilon_{\mathrm{fb}}=\frac{C_{1}}{\lambda^{2}} \frac{n_{\mathrm{e}} n_{\mathrm{i}}}{\sqrt{T_{\mathrm{e}}}} \frac{g_{1,1}}{U_{1}} \xi_{\mathrm{fb}}\left(\lambda, T_{\mathrm{e}}\right)\left(1-\mathrm{e}^{\left.-h c / \lambda k T_{\mathrm{e}}\right)} .\right.
\end{gathered}
$$

The symbols in equations (8)-(11) are shown in table 1 . Below $15000 \mathrm{~K}$, the number of doubly ionized argon ions is negligible. Also the excited ion state density is small

Table 1. The symbols used in equations (4)-(7).

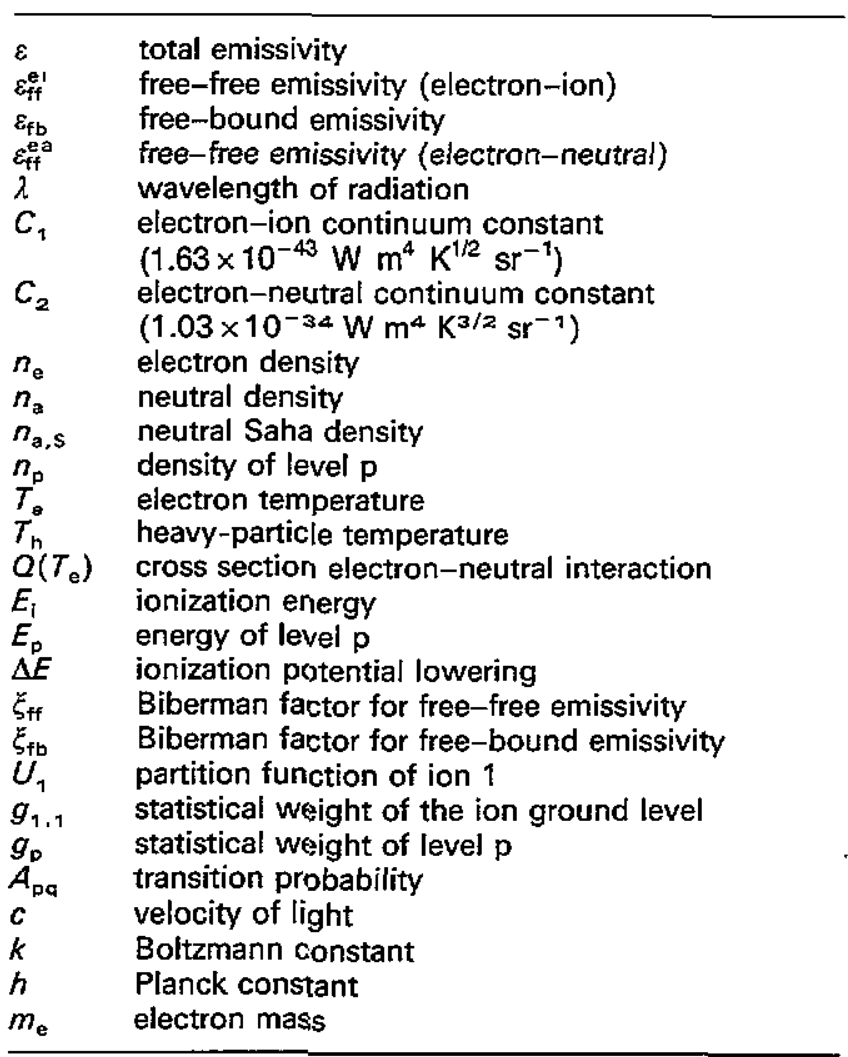

relative to the ion ground state density, $g_{1,1} \simeq U_{1}=4+2$ $\exp \left(-2059 / T_{\mathrm{e}}\right) \simeq 6$. The ground state density of singly ionized ions is then equal to the electron density. More information on the continuum emissivity of argon may be found in $[12,13]$ and references therein. For electron temperatures higher than $10000 \mathrm{~K}$ the integrated freefree electron-atom emissivity is less than $2 \%$ of the integrated free-bound plus free-free electron-ion emissivity, and decreases rapidly with increasing temperature as can be seen in figure 3 [14].

Therefore the term $\varepsilon_{f f}^{\text {ea }}$ in equation (10) is omitted in the calculations of the continuum emissivity. Note that in this case the expression for the continuum emission is also valid for non-LTE situations. The total continuum emissivity can be approximated assuming quasineutrality and a temperature of about $12000 \mathrm{~K}$ by [12]:

$$
\varepsilon_{\text {cont }}=C_{1} \frac{n_{\mathrm{e}}^{2}}{\lambda^{2} \sqrt{T_{\mathrm{e}}}}
$$

The line/continuum ratio provides the electron temperature using the equation

$$
T_{\mathrm{e}}=\frac{E_{\mathrm{p}}}{k} \ln ^{-1}\left(\left.\frac{I_{\text {cont }}}{I_{\text {line }}}\right|_{\text {top }} \frac{A_{\mathrm{pq}} h c \lambda g_{\mathrm{p}}^{\| \prime} \sqrt{T_{\mathrm{e}}}}{4 \pi U_{1} \beta_{\mathrm{p}}^{+} C_{1} \gamma_{\mathrm{p}} n_{\mathrm{e}}}\right)
$$

where $U_{1}$ is the partition function of the ArII system [15], $g_{\mathrm{p}}^{\|}$the statistical weight of the upper level and $E_{\mathrm{p}}$ the energy difference between the upper level $p$ and the ion ground state; $C_{1}=1.63 \times 10^{-43} \mathrm{Wm}^{4} \mathrm{~K}^{1 / 2} \mathrm{sr}^{-1}$ is a constant, $n_{\mathrm{e}}$ is the electron density, $A_{\mathrm{pq}}$ is the transition probability from level $\mathrm{p}$ to $\mathrm{q}$ for spontaneous emission, and $\gamma_{p}$ is the FWHM of the line profile. Equation (13) is analysed assuming LTE conditions in the ion state if the over(under)population factor of the ion ground state $\beta_{\mathrm{p}}^{+}=1$. More likely is a slight underpopulation of the excited level relative to the ion ground state. This effect can be accounted for by an underpopulation factor $\beta_{\mathrm{p}}^{+}<1$; then the argument of the logarithm in equation (9) has to be multiplied by $\left(\beta_{p}^{+}\right)^{-1}$, as has been done. The factor $\beta_{\mathrm{p}}$ in our plasma will be about 1 [16].

An alternative simple and fast method to check the electron temperature is a method using the electrical

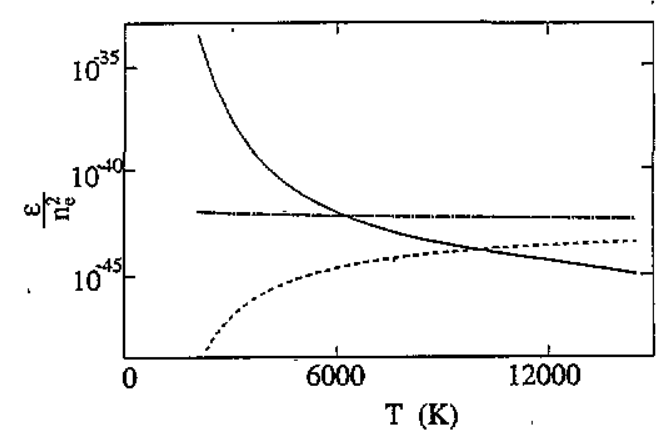

Figure 3. Continuum emissivities of an argon plasma at $4 \times 10^{4} \mathrm{~Pa}$ for a wavelength of $435.4 \mathrm{~nm}$ with $\Delta \lambda=0.04 \mathrm{~nm}$ weighted by the square of the electron density: ( $\longrightarrow$ ): free-free electron-atom radiation; (---): free-free electron-ion radiation; and $(-\cdot-)$ : free-bound radiation. 
conductivity of the plasma in the arc channel as a function of electron temperature according to Frost [17] as used by Kroesen [1]. The experimental value of the conductivity is obtained assuming a homogeneous current distribution over the arc channel cross section with an effective radius $R_{\text {eff }}$ :

$$
\sigma_{\exp }=I d /\left(\pi R_{\mathrm{eff}}^{2} \Delta V\right) \sim T_{\mathrm{e}}^{3 / 2}
$$

where $I$ is the arc current, $d$ the distance between two adjacent cascaded plates, $R_{\text {eff }}$ the effective arc channel radius $(0.9$ times the channel radius, for a non-flowing arc, according to Timmermans [6]), and $\Delta V$ is the potential difference between two adjacent plates. Because the plate potentials can easily be measured outside the arc, this method is very fast for obtaining the electron temperature. It contains, however, the uncertainty of the effective arc channel radius $R_{\text {eff }}$.

\section{Results}

In the experiments an argon ion line $(435.4 \mathrm{~nm}$, of the $4 \mathrm{p}-4 \mathrm{~d}$ multiplet is used because the Stark broadening of ion lines is about a factor of ten smaller than for neutral lines [10]. For our plasma this means that, for an argon ion line, the Stark width and the Doppler width are of the same order of magnitue. The measured profiles are perfectly symmetric and the width is of the order of $5 \mathrm{pm}$ which consists of the Gaussian apparatus profile and about equal parts of Doppler and Lorentz contributions. After deconvolution of the line profiles of the Ar II line $(435.4 \mathrm{~nm})$ the Gaussian part gives the heavy-particle temperature $T_{\mathrm{h}}$. The reproducibility of the measured temperature is about $2 \%$. Note that the measured temperatures (and electron densities) are not values at the axis of the arc, but represent an average over the cross section, weighted with the detection efficiency and the density of the radiating particles, as a function of the radial position. Nevertheless, these values are close to the axial ones because of the maximum values of ion and electron densities and corresponding line intensity, and a higher detection efficiency for the centre.

In figure 4 the heavy-particle temperature is plotted

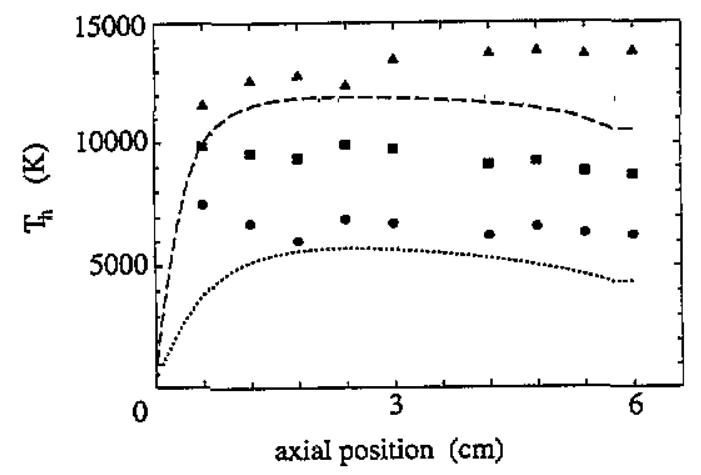

Figure 4. Variation of the heavy-particle temperature along the arc for different arc currents at an argon flow rate of 50 scc $\mathrm{s}^{-1}$. The lines are model calculations: $(\mathbf{A},-----)$ : $50 \mathrm{~A}$; (a): $30 \mathrm{~A}(\mathrm{O} . \cdots): 2.0 \mathrm{~A}$. as a function of axial position for a constant argon flow rate of $50 \mathrm{sccm} \mathrm{s} ~^{-1}$ and three different arc currents. As can be expected, the temperature increases with increasing arc power. The experimental values are slightly higher than the model predictions. Note, however, that especially for lower arc currents, the model values approach the maximum temperature slowly relative to the experimental temperatures. This effect points to a too slow 'energy' coupling between the argon heavy particles and the electrons in the model. The heavy-particle temperatures predicted by the model are therefore also lower than the measured temperatures.

Lowering the argon flow rates gives (figure 5) a different behaviour of the heavy-particle temperature. One would expect a higher temperature for lower flows. The heating at the cathode side is faster for low flows but does not show a clear increase as a function of axial position. The expansion terms in the energy equations plus the heat escaping through the arc walls are, for lower flows, and thus lower plasma pressure, dominant over the energy input through electron-heavy-particle collisions. Efficiency measurements by de Regt [18] and de Graaf [19], who measured the escaping energy through the water cooling, show a significantly lower power efficiency, which is the net power flowing out of the arc at the anode side, for lower gas flow rates. For rates ranging between 30 and $100 \mathrm{scc} \mathrm{s} \mathrm{s}^{-1}$ the energy efficiency increased from $50 \%$ to $70 \%$. At higher arc currents the effect shown in figure 5 diminishes and we see in figure 6 that now for lower gas flow rates the heavy-particle temperature increases, although this is not predicted by the model. The heating of the heavy particles to a maximum value about halfway along the arc is seen in both the model values and the experimental values. For gas flow rates higher than $100 \mathrm{scc} \mathrm{s}^{-1}$ the scatter in the experimental temperatures is increasing due to instabilities in the arc. The $100 \mathrm{scc} \mathrm{s}^{-1}$ setting was about the maximum gas flow rate at which we can measure the heavy-particle temperature.

For low gas flow rates and low arc currents, we see in the experimental values in figure 7 again fast heating and in the model far too slow coupling between electrons and heavy particles. Both the model and the experiments

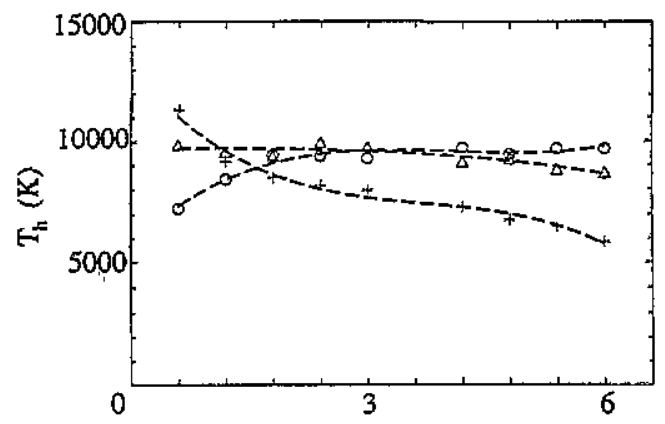

Figure 5. The heavy-particle temperature as a function of axial position for different argon flow rates $\left(+, 20 \mathrm{scc} \mathrm{s}^{-1}\right.$; $\triangle, 50 \mathrm{scc} \mathrm{s}^{-1}$, and $\left.0,100 \mathrm{scc} \mathrm{s}^{-1}\right)$ at an arc current of $30 \mathrm{~A}$. The lines through the points are polynomial fits to show the trend. 


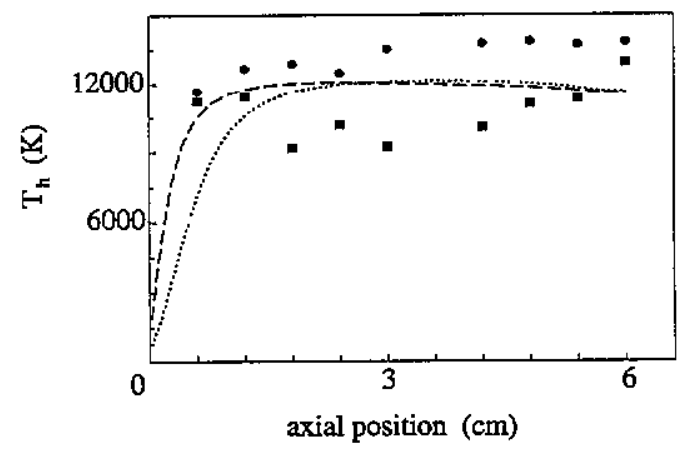

Figure 6. Variation of the heavy-particle temperature versus axial position for $50 \mathrm{~A}$ arc current: $(0,--): 50 \mathrm{scc} \mathrm{s}^{-1}$; $(\mathrm{A}, \cdots): 100 \mathrm{scc} \mathrm{s}^{-1}$.

show that, for low power and flow settings, the heavyparticle temperature decreases monotonically (after $2 \mathrm{~cm}$ of arc length).

\subsection{Alternative energy coupling between electrons and heavy particles}

In this section we propose and estimate the additional energy transfer between the electrons and heavy particles in (sub)atmospheric plasmas by termolecular ion formation and dissociative recombination. In most models of the dynamics of high-temperature plasmas, like ICPS (inductively coupled plasmas), and thermal arcs, only the electron-neutral and ion heat transfer in elastic collisions is taken into account. In comparing the calculated evaluation of the heavy-particle temperature in a flowing plasma with measured data, it has become apparent that a heat transfer mechanism is missing. (See for example figures 4,6 and 7). From the model calculations it can be concluded that a length of about $2 \times 10^{-2} \mathrm{~m}$ is needed for coupling of the heavy-particle temperature $T_{\mathrm{h}}$ and electron temperature $T_{\mathrm{e}}$ through elastic collisions; this is equivalent to a time constant of $10^{-4} \mathrm{~s}$ $\left(2 \times 10^{-2}(\mathrm{~m}) / 200\left(\mathrm{~m} \mathrm{~s}^{-1}\right)\right.$. In the experiment this time constant is approximately ten times smaller. Also, calculations for an ICP indicate a difference between model and experiment, and it is useful to consider other candidates for electron-heavy-particle energy transfer [20]. A possible candidate would be energy transfer from

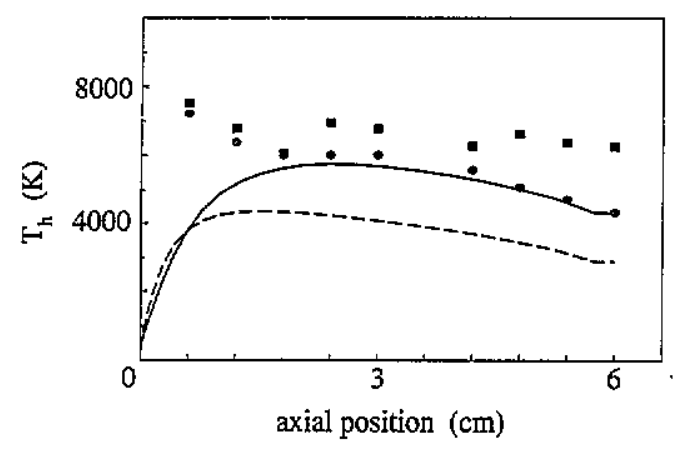

Figure 7. Heavy-particle temperature versus axial position for low arc current (20 A) and low argon flow rates: $(\mathbb{B},-)$ : $50 \operatorname{scc~s}^{-1} ;(0,--)$ : $20 \operatorname{scc~s}^{-1}$. internal energy (excitation/ionization), originating from electron energy, to heavy-particle energy, through termolecular association followed by dissociative recombination of the resulting molecular ion and subsequent electronic re-ionization as shown schematically in figure 8 . In this process the difference between the atomic ionization energy and the energy of the level at which the recombination ends is dissipated in translational energy of the resulting neutrals. In order to estimate the effectiveness of the process, we will discuss in detail the three steps in this process: termolecular association, dissociative recombination and electronic re-ionization of the resulting level.

5.1.1. Termolecular association. The process $\mathrm{Ar}^{+}+$ $\mathrm{Ar}+\mathrm{M} \rightarrow \mathrm{Ar}_{2}^{+}+\mathrm{M}$, also called ion conversion, has received considerable attention in the earlier literature on excimer lasers and on afterglow plasmas. Quoted rate constants for ion conversion in room temperature gas are $\beta_{\mathrm{He}}=0.8 \times 10^{-43} \mathrm{~m}^{6} \mathrm{~s}^{-1}$ when $\mathrm{M}$ is helium [21], $\beta_{\mathrm{Ar}}=2.26 \times 10^{-43} \mathrm{~m}^{6} \mathrm{~s}^{-1}$ when $M$ is argon, and $\beta_{\mathrm{Ne}}=0.47 \times 10^{-43} \mathrm{~m}^{6} \mathrm{~s}^{-1}$ when $\mathrm{M}$ is neon [22-24]. The temperature dependence can be estimated from the $E / p$ dependence of the similar process in neon found by Dielis [24]. By working out the relation between $E / p$ and ion energy, a $T^{-0.5}$ dependence can be deduced for energies $\gg 0.3 \mathrm{eV}$, and a roughly constant value for lower temperatures. As an approximation we will assume the following rate coefficient for argon:

$$
k_{\mathrm{c}} \simeq 2.26 \times 10^{-43} \mathrm{e}^{-\hat{T}_{h} / 1}
$$

where $\hat{T}_{\mathrm{h}}$ denotes the temperature in electron-volts. (Without the accent ${ }^{-}$the temperatures are given in kelvin.)

The inverse process could also be of importance, in particular for higher temperatures (and energies): $\mathrm{Ar}_{2}^{+}+\mathrm{Ar} \rightarrow \mathrm{Ar}^{+}+\mathrm{Ar}+\mathrm{Ar}$. The rates have to be compared with those of dissociative recombination. For neon, rates found by Dielis [24] are evaluated. For argon

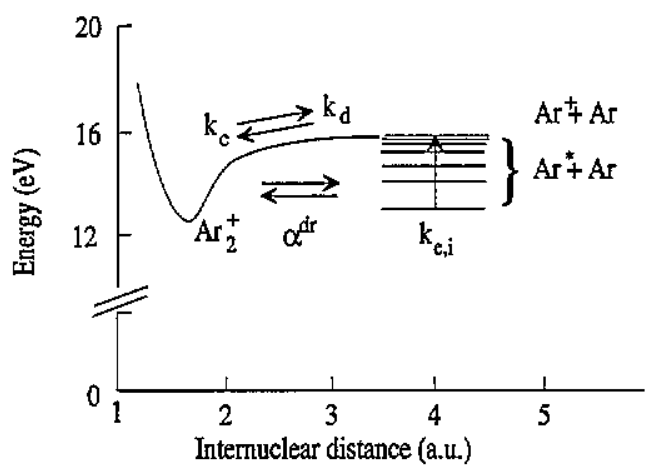

Figure 8. Energy diagram for $\mathrm{Ar}_{2}^{+}$(schematically). The processes plotted in the figure are termolecular association $\left(k_{c}\right)$; dissociation $\left(k_{\mathrm{d}}\right)$; dissociative recombination $\left(\alpha^{\mathrm{dr}}\right)$ leading to $\mathrm{Ar}^{*}+\mathrm{Ar}$; and electronic excitation (ionization) of $\mathrm{Ar}^{*}$. The lowest $\mathrm{Ar}^{*}$ level coincides with the argon $4 \mathrm{p}$ level at $13.3 \mathrm{eV}$. 
the rate is expected to be 4-5 times that of neon and can be represented in the energy region $0.1-1 \mathrm{eV}$ by

$$
k_{\text {diss }} \simeq 5 \times 10^{-17} \hat{T}_{\mathrm{i}}^{3.5}
$$

and would be in the range $10^{-19}-10^{-17} \mathrm{~m}^{3} \mathrm{~s}^{-1}$ for energies $0.1-1 \mathrm{eV}$.

5.1.2. Dissociative recombination. Hasted [25] gives two different curves, as obtained by various authors up to 1972. The first, valid for afterglow plasmas, is roughly characterized by

$$
\alpha^{\mathrm{dr}} \simeq 10^{-13} / \sqrt{ } \hat{T}_{\mathrm{c}} \mathrm{m}^{3} \mathrm{~s}^{-1}
$$

and the second (obtained from shock experiments and also dependent on the gas temperature) is $\alpha^{\mathrm{dr}} \simeq 2 \times 10^{-15} / \hat{T}_{\mathrm{e}}^{1.5}\left(\mathrm{~m}^{3} \mathrm{~s}^{-1}\right)$, which would be about 50 times smaller for $\hat{T}_{\mathrm{c}}=\hat{T}_{\mathrm{g}}=1 \mathrm{eV}$. At $\hat{T}_{\mathrm{e}}=\widehat{T}_{\mathrm{g}}=0.1 \mathrm{eV}$ the difference would be a factor of five. We suspect that this (shock tube) result is less applicable as it is probably influenced by the inverse of termolecular association. The competing process of dissociation of ions leads to less molecular ion formation and for that reason less recombination. It would also explain the heavy-particle temperature dependence, which is otherwise difficult to understand. Later Shiu and Biondi [26] obtained a rate represented by

$$
\alpha^{\mathrm{dr}} \simeq 9.1 \times 10^{-13}\left(T_{c} / 300\right)^{-0.61}
$$

which differs by only up to $25 \%$ from values from equation (17). For convenience equation (17) is used to compare dissociative recombination and dissociation of molecular ions:

$$
n_{0} k_{\text {diss }}: k_{\text {dr }} n_{e} \simeq 5 \times 10^{-4} \hat{T}_{e}^{4}\left(n_{0} / n_{e}\right)
$$

It is clear that for $n_{\mathrm{e}} / n_{0}>10^{-6}$ at low $T_{\mathrm{e}}$ and $T_{\mathrm{l}}$ and for $n_{e} / n_{0}>10^{-3}$ at $\hat{T}_{\mathrm{e}}=\widehat{T}_{\mathrm{i}}=1 \mathrm{eV}$, dissociative recombination governs the kinetics. Hence dissociation can be ignored.

5.1.3. Re-ionization. There are strong indications (from work by Frommhold [27] and Veatch [21]) that the dissociative recombination process ends in one (or more) of the $4 \mathrm{p}$ levels of argon, such as the upper level of the $696.5 \mathrm{~nm}$ line, the $4 \mathrm{p}^{\prime}[1 / 2]$ level at $13.33 \mathrm{eV}$. Hence the $4 \mathrm{p}$ excitation and ionization rates apply. In argon models, rates of the order of $10^{-12} \mathrm{~m}^{3} \mathrm{~s}^{-1}$ are assumed for
$\widehat{T}_{\mathrm{e}} \simeq 1 \mathrm{eV}$. Hence for $n_{\mathrm{e}} \geqslant 10^{20} \mathrm{~m}^{-3}$ the produced excited neutral is re-ionized, and in terms of mass balance nothing has happened. In one circular chain of reactions, the electron population lost about $2.5 \mathrm{eV}$ in re-ionizing the $4 p$ state neutral and the neutral population gained about $2.5 \mathrm{eV}$ in translational energy in processes of dissociative recombination and electronic re-ionization. (See again figure 8, where the reaction chain is schematically represented.) Hence as an addition to the regular energy transfer through elastic collisions, an additional energy transfer appears:

$$
Q_{3 \mathrm{~d}}=2.26 \times 10^{-43} \mathrm{e}^{-\hat{T}_{h} / 1} n_{0}^{2} n_{\mathrm{c}} \times 2.5 \times 1.6 \times 10^{-19} .
$$

Here it is assumed that termolecular association is the rate-limiting step. As a consequence $n_{\mathrm{Ar}^{+}} \gg n_{\mathrm{Ar}^{2+}}$, since dissociative recombination is fast relative to termolecular ion conversion. Then, the energy lost is the rate for ion conversion multiplied by the energy transfer per cycle of $2.5 \mathrm{eV}: 0.9 \times 10^{-61} \exp \left(-\hat{T}_{\mathrm{h}}\right) n_{0}^{2} n_{\mathrm{c}} \mathrm{W} \mathrm{m}^{-3}$. For $n_{0}=10^{24}$ $\mathrm{m}^{-3}, n_{\mathrm{c}}=10^{22} \mathrm{~m}^{-3}, \hat{T}_{\mathrm{h}}=0.3 \mathrm{eV}$, this leads to a threeparticle heat transfer $Q_{3 \mathrm{~d}}=6.6 \times 10^{8} \mathrm{~W} \mathrm{~m}^{-3}$. The traditionally used transfer rate, $Q_{\mathrm{ch}}=3 n_{\mathrm{e}}\left(m_{\mathrm{e}} / m_{\mathrm{h}}\right) v_{\mathrm{eh}} k\left(T_{\mathrm{c}}-T_{\mathrm{h}}\right)$, gives, for these parameters, a rate of $4.5 \times 10^{8} \mathrm{~W} \mathrm{~m}^{-3}$ (using $v_{\text {eh }}=10^{10} \mathrm{~s}^{-1}$ and $\Delta T=8000 \mathrm{~K}$ ). The smaller the temperature non-equilibrium, the smaller $Q_{\mathrm{eh}}$, leading to an extra energy transfer $Q_{3 \mathrm{~d}}$ of the order of twice $Q_{\mathrm{eh}}$. The proposed scheme to improve the model calculations, via inclusion of the additional electron-heavy-particle heat transfer through the production of $\mathrm{Ar}_{2}^{+}$in the highpressure region, has been implemented in the model. It seems not to be sufficient to explain the additional heating of the heavy particles, as can be seen in figure 9 . Assuming a second production cycle through two argon ions and an argon neutral, $\mathrm{Ar}^{+}+\mathrm{Ar}+\mathrm{Ar}^{+} \rightarrow \mathrm{Ar}_{2}^{+}+\mathrm{Ar}^{+}$, the energy transfer rate is assumed to increase with a factor

$$
1+\left(n_{\mathrm{c}} / n_{\mathrm{o}}\right)\left(k_{\mathrm{ii}} / k_{\mathrm{io}}\right)
$$

where $k_{\mathrm{il}}$ and $k_{\mathrm{i} 0}$ denote ion-ion and ion-neutral collision rates of coefficients $[28,29]$. Preliminary calculations by van den Bercken show that the extra factor increases the energy transfer up to a $30 \%$ faster coupling between electrons and heavy particles [30]. Further research is still needed to understand the effects of energy coupling through $\mathrm{Ar}_{2}^{+}$production cycles.

In figure 10 the electron density, obtained from the Lorentz part of the line profile, is plotted as a function of

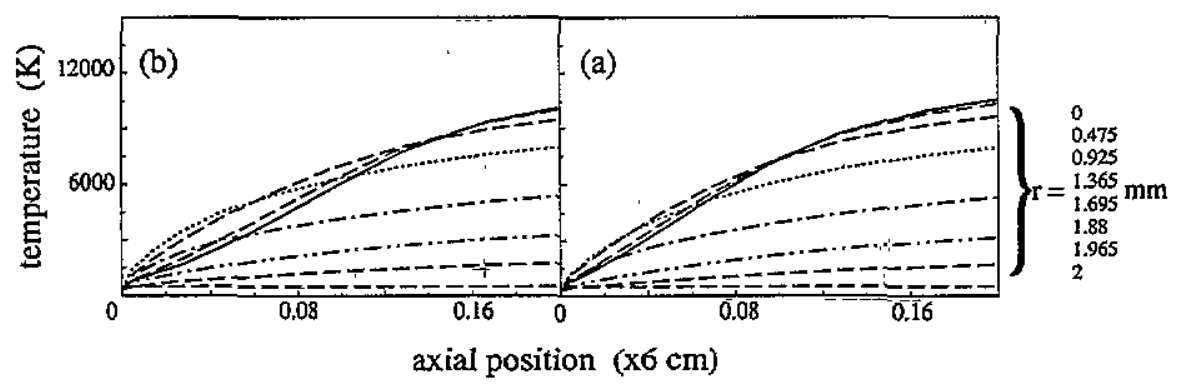

Figure 9. Heavy-particle temperature versus axial position with (a) and without (b) the $\mathrm{Ar}_{2}^{+}$cycle incorporated in the model calculations. 


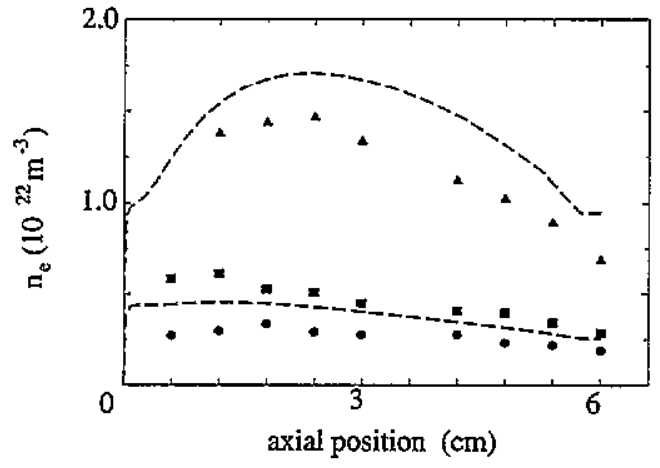

Figure 10. Electron density variation along the axis for an argon flow rate of $50 \mathrm{scc} \mathrm{s}^{-1}$ and different arc currents: $(\triangle)$ : $50 \mathrm{~A} ;(0): 20 \mathrm{~A}$. The broken lines are model calculations for $50 \mathrm{~A}$ and $20 \mathrm{~A}$ arc current.

axial position and arc current for a gas flow rate of 50 $\mathrm{sccm} \mathrm{s}{ }^{-1}$. The electron density increases as a function of arc current, as shown by the model and experimental electron densities. Especially for higher currents, we see that a maximum appears in the electron density about halfway along the arc channel. This is caused by the almost linearly increasing velocity (and decreasing plasma pressure), as a function of axial position, while the total particle flux is constant [5]. This does not, however, mean that electrons are lost, because the ionization degree according to the model still increases [10]. The model values at the axis are up to $20 \%$ larger than the experimental values. Here we have to take into account the fact that the measured electron density is an average value over the cross section weighted by the detection volume, resulting in a value between the (model) average and the density at the arc channel axis [5]. The values for the electron density are in good agreement with measurements by Kroesen [1], who used $\mathrm{H}_{\beta}$ broadening of seeded hydrogen 'impurities' in the arc.

For lower argon flow rates and low currents, figure 11 shows that the maximum in the electron density is reached increasingly far upstream in the arc channel (closer to the arc cathode). In figure 12 the model calculations also show a shift of the maximum towards the channel entrance. This is consistent with the temperature measurements, which show that at low gas flow

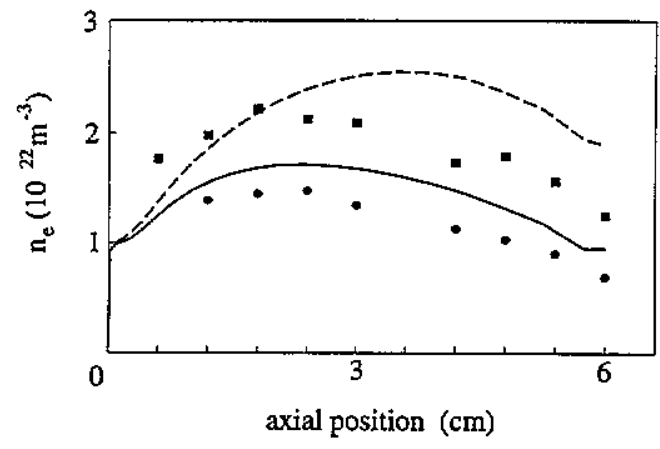

Figure 11. Variation of electron density along the arc axis for different argon flow rates at $50 \mathrm{~A}$ arc current: (.,--): 100 scc s ${ }^{-1} ;(\theta,-): 50 \operatorname{scc~s}^{-1}$.

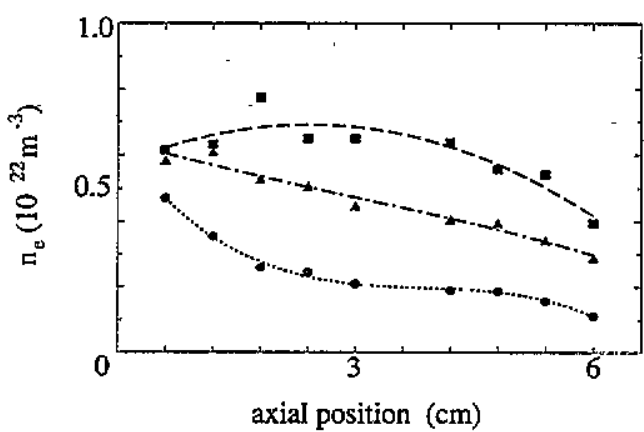

Figure 12. Electron density versus axial position at a current of $30 \mathrm{~A}$ and argon flow rates of 100,50 and $20 \mathrm{scc} \mathrm{s}^{-1}$. The curves represent polynomial fits to the experimental data: $(\mathrm{a},--): 100 \operatorname{scc~s}^{-1} ;(\triangle,-\cdot-): 50 \operatorname{scc~s}^{-1} ;(0, \cdots)$ : $20 \operatorname{scc} s^{-1}$.

rates the gas is heated already in the first few $1-2 \mathrm{~cm}$ of the arc.

Decreasing the arc current leads to the same behaviour of the electron density along the arc, as is shown in figure 13, where the model also predicts the same behaviour for a gas flow rate of $20 \mathrm{scc} \mathrm{s}^{-1}$.

In figure 14 are plotted the electron temperatures which are obtained by the line/continuum ratio (closed symbols) and by the 'conductivity' method. The absolute values of the temperatures obtained by the Fabry-Pérot line/continuum ratio method are slightly $(20 \%)$ higher than those predicted by the model [5]. The reason for this discrepancy is unknown. We should note, however, that equation (12) is an oversimplification, especially for low electron temperatures where free-free electron-atom radiation also adds to the theoretical emissivity, leading to a too high electron temperature when this term is not taken into account. The measured and modelled temperatures show the same qualitative dependence on the axial position. Only a very slight increase of electron temperature can be observed. The same behaviour is shown by the 'conductivity' method (open symbols). The electron temperature increases as a function of arc power and argon flow rate, which is consistent with the model. The 'conductivity' temperatures increase a little more strongIy as a function of the axial position, probably because of the rather arbitrary value of the effective plasma channel

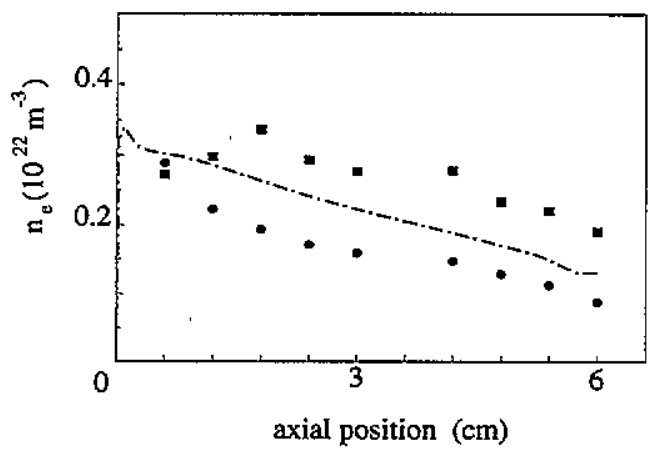

Figure 13. Electron density variation along the arc channel for low flow rates (50 and $20 \mathrm{scc} \mathrm{s}^{-1}$ ) and low arc current (20 A): $(\square): 50 \operatorname{scc~s}^{-1}$ and $(0, \ldots \cdot-): 20 \mathrm{scc} \mathrm{s}^{-1}$. 


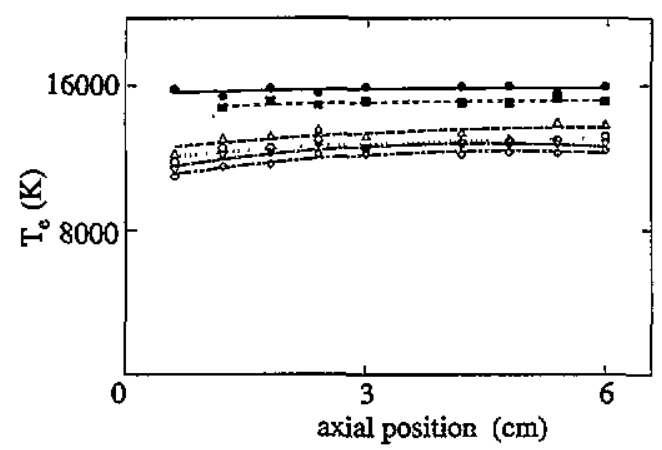

Figure 14. Electron temperature along the arc for a $50 \mathrm{~A}$ arc current at several argon flow rates. The open symbols represent temperatures calculated by the 'conductivity' method, and the closed symbols are calculated using the line/continuum ratio: $(\square)$ : $100 \mathrm{scc} \mathrm{s}^{-1} ;(0): 50 \mathrm{scc} \mathrm{s}^{-1} ;(\triangle)$ : $20 \operatorname{scc~s}^{-1} ;(0): 40 \operatorname{scc~s}^{-1} ;(): 60 \operatorname{scc~s}^{-1} ;(\diamond): 100$ scc $s^{-1}$.

radius $R$. We took a value of 0.9 , as is shown to be correct for stationary arcs by Timmermans [6], for every axial position. Assuming a smaller radius near the cathode, which increases up to 0.9 downstream, will result in the calculated electron temperature. This then results in a flatter electron temperature profile than for the line/continuum method and the model predictions. The absolute values of the temperatures obtained by the 'conductivity' method are very close to the model values ( $<5 \%$ difference).

\section{Summary and conclusions}

The Fabry-Pérot interferometry diagnostic used is shown to be a very good tool for measuring temperatures and electron densities in a strongly flowing cascaded arc, resulting in an accuracy of about $2-5 \%$. Only one line profile needs to be measured to obtain $T_{\mathrm{e}}, T_{\mathrm{h}}$ and $n_{\mathrm{e}}$. The numerical results are already quite close to the experimental ones and can be used to predict the behaviour of the arc plasma.

The strongly flowing cascaded arc plasma shows a strong temperature disequilibrium, especially for low arc currents and argon flow rates. These measurements validate the necessity of a two-temperature twodimensional computer model. They can also lead to an improvement of the description of the physical (plasma) processes to be incorporated in the model.

Further research needs to be done to close the gap between experimental data and the model. The model can be further improved, for example, by including radiation transport in a more refined way and by using a two-dimensional current density, and thus electric field. The latter can possibly lead to an apparently faster coupling between the electron and heavy-particle temperature, because the actual plasma channel near the cathode may be substantially narrower than further downstream. The measured temperature near the cathode will then be higher and thus closer to the electron temperature. The model as it is now, however, spreads the energy over the full cross section, leading to a lower modelled temperature. The 'conductivity' measurements at the arc entrance also point to an effectively smaller diameter of the plasma channel.

Finally, a summary of results is presented in figure 15 where trends are given for the plasma parameters at the arc exit. As a function of arc power and argon flow rate the electron temperature, heavy-particle temperature, electron density, degree of ionization, and plasma pressure at the exit of the cascaded arc are plotted. The plotted results are obtained both experimentally and theoretically.

In figures $15(a)$ and $(b)$ we see that the electron temperature varies only by about $10 \%$ for the reactor settings used. From figures $15(c)$ and $(d)$ we see that the heavy-particle temperature increases linearly with arc current, except for low gas flow rates. At low flow rates (about $20 \mathrm{scc} \mathrm{s}^{-1}$ ) $T_{\mathrm{h}}$ is constant. At constant arc currents we notice that the heavy-particle temperature is nearly constant. The pressure at the nozzle throat increases approximately linearly with power and with argon flow rate as can be seen in figures $15(e)$ and $(f)$. The most important parameter is the electron density, or rather the degree of ionization. The degree of ionization is directly related to the number of electrons and ions (per second) that emerge from the arc nozzle. This number

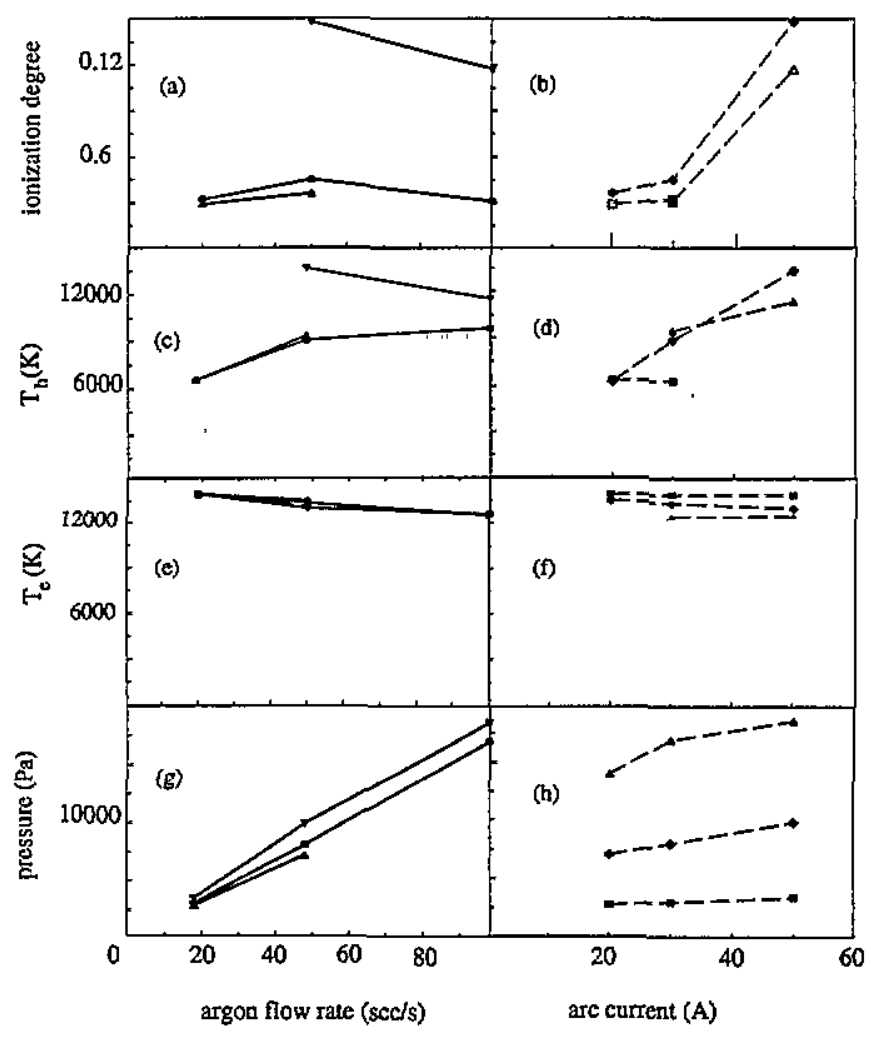

Figure 15. Here are shown $(a)$ and $(b)$ ionization degree; $(c)$ and $(d)$ heavy-particle temperature; $(e)$ and $(f)$ electron temperature; and $(g)$ and $(h)$ plasma pressure versus argon flow rate and arc current respectively: $(\Delta)$ : Ar flow rate variation for $l_{\text {arc }}=20 \mathrm{~A} ;(0)$ : Ar flow rate variation for $l_{\text {arc }}=30$ $A ;(\nabla)$ : Ar flow rate variation for $I_{\text {arc }}=50 A_{i}\left(\Pi_{m}\right)$ : arc current variation for $[\mathrm{Ar}]=20 \mathrm{scc} \mathrm{s}^{-1} ;(\Delta)$ : arc current variation for $[\mathrm{Ar}]=50 \mathrm{scc} \mathrm{s}^{-\mathrm{t}} ;(\triangle)$ : arc current variation for $[\mathrm{Ar}]=100 \mathrm{scc}$ $s^{-1}$. 
then is directly related to the degree of dissociation and ionization of the monomers injected in the nozzle. In figures $15(\mathrm{~g})$ and $(h)$ we clearly see that at constant argon flow rates the degree of ionization increases more than linearly with arc current (power). For constant arc current and variable flow rates, we notice some kind of optimum flow rate for which the degree of ionization is maximum, although the variation in degree of ionization is small.

This summary of results for the emerging plasma beam at the throat of the arc nozzle gives us an estimation of the boundary conditions in the nozzle, at the position where hydrocarbons are injected. At that point we can start modelling the relation between the external reactor parameters and the plasma (composition) parameters in the expansion zone.

\section{Acknowledgments}

This work is part of the research programme of the Stichting voor Fundamenteel Onderzoek der Materie (FOM).

\section{References}

[1] Kroesen G M W, Schram D C and de Haas C M 1990 Plasma Chem. Plasma Proc. 10531

[2] Bachmann P K, Beulens J J, Kroesen G M W, Lydtin H, Schram D C and Wiechert D U 1990 Proc. Surface Modification Technologies III ed T S Sudarshan and D G Bhat (Warrendale: The Minerals, Metals \& Materials Society) p 69

[3] Savvides N and Window B $1985 \mathrm{~J}$. Vac. Sci. Technol. A3 2386

[4] Savvides N 1986 J. Appl. Phys. 594133

[5] Beulens J J, Milojevic D, Schram D C and Vallinga P M 1991 Phys. Fluids B 32548

[6] Timmermans C J, Rosado R J and Schram D C 1985 Z. Naturf. 40A 810
[7] de Haas J C M 1986 Ph D Thesis Eindhoven University of Technology, The Netherlands

[8] Mostaghimi J, Proulx P and Boulos M I 1985 Numerical Heat Transfer 8187

[9] Mostaghimi J, Proulx P and Boulos M I 1987 J. Appl. Phys. 611753

[10] Griem H R 1974 Spectral Line Broadening by Plasmas (New York: Academic)

[11] Sobel'man I I 1972 Introduction to the Theory of Atomic Spectra (Oxford: Pergamon)

[12] Wilbers A T M, Kroesen G M W, Timmermans C J and Schram D C 1991 J. Quant. Spectrosc. Radiat. Transfer 451

[13] Wilbers A T M and Schram D C $1991 \mathrm{~J}$. Quant. Spectrosc. Radiat. Transfer 46299

[14] Wilbers A T M, Beulens I $J$ and Schram D C 1991 J. Quant. Spectrosc. Radiat. Transfer 46385

[15] Drawin H W and Felenbok P 1965 Data for Plasmas in LTE (Paris: Gauthier-Villars)

[16] Vaessen P H M 1984 PhD Thesis Eindhoven University of Technology, The Netherlands

[17] Frost L S 1961 J. Appl. Phys. 322029

[18] de Regt J M 1989 private communication, Eindhoven University of Technology, The Netherlands

[19] de Graaf M J 1989 private communications, Eindhoven University of Technology, The Netherlands

[20] Benoy D 1989 private communications, Eindhoven University of Technology, The Netherlands

[21] Veatch G E and Oskam H J 1970 Phys. Rev. Al 1498

[22] Liu W-C and Conway D C 1975 J. Chem. Phys. 62 3070

[23] Sugawara M, Okada T, Kobayashi Y 1986 J. Phys. D: Appl. Phys. 191213

[24] Dielis J 1979 PhD Thesis Eindhoven University of Technology, The Netherlands

[25] Hasted J B 1972 Physics of Atomic Collisions (London: Butterworth)

[26] Shiu Y J and Biondi M I 1978 Phys. Rev. A 17868

[27] Frommhold L and Biondi M I 1969 Phys. Rev. 185244

[28] Timmermans C J 1984 PhD Thesis Eindhoven University of Technology, The Netherlands

[29] Braginskii S I 1965 Rev. Plasma Phys. 1205

[30] van den Bercken R 1991 private communication, Eindhoven University of Technology, The Netherlands 